\title{
Impact of a teaching program on outcome quality of white light transurethral resection for bladder tumor: A cohort study
}

Rodolfo Hurle ${ }^{1}$, Roberto Peschechera ${ }^{1}$, Nicolò Maria Buffi ${ }^{1}$, Giovanni Lughezzani ${ }^{1}$, Emanuela Morenghi ${ }^{2}$, Alberto Saita ${ }^{1}$, Luisa Pasini ${ }^{1}$, Paolo Casale ${ }^{1}$, Mauro Seveso ${ }^{1}$, Silvia Zandegiacomo ${ }^{1}$, Gianluigi Taverna ${ }^{1}$, Alessio Benetti ${ }^{1}$, Ivano Vavassori ${ }^{1}$, Piergiuseppe Colombo ${ }^{3}$, Massimo Lazzeri*1 ${ }^{*}$, Giorgio Guazzoni ${ }^{1,2,3}$

${ }^{1}$ Department of Urology, Istituto Clinico Humanitas IRCCS, Clinical and Research Center, Rozzano (Milan), Italy

${ }^{2}$ Biostatistics Unit, Istituto Clinico Humanitas IRCCS, Clinical and Research Center, Rozzano (Milan), Italy

${ }^{3}$ Department of Pathology, Istituto Clinico Humanitas IRCCS, Clinical and Research Center, Rozzano (Milan), Italy

Received: May 26, 2016

DOI: $10.5430 /$ jst.v6n2p56
Accepted: June 29, 2016

Online Published: July 11, 2016

\begin{abstract}
Objective: To test the hypothesis that a teaching program improves the quality of transurethral resection of bladder tumor (TURBT) and decreases the risk of early recurrence.

Material and methods: This is an observational retrospective cohort study of prospectively recorded data of patients with first clinical diagnosis of non-muscle-invasive bladder cancer (NMIBC), scheduled for TURBT. In 2005 a systematic TURBT teaching program was introduced in our Department. We reviewed the charts of patients who underwent TURBT in the years 1998-2004, when no tutoring was applied, and those who underwent TURBT in the years 2005-2010. The outcomes of interest were: presence/absence of detrusor muscle (DM), carcinoma in situ (CIS) detection, complication rate and recurrence rate at the first follow-up cystoscopy (RRFF-C).

Results: Complete data from 427 patients were available: 199 before and 228 after the introduction of the teaching program. Multivariable logistic analysis showed that the training program was an independent prognostic factor for DM (presence) rate $(\mathrm{OR}=3.92,95 \% \mathrm{CI}=2.42-6.33), \mathrm{CIS}$ detection rate $(\mathrm{OR}=4.36,95 \% \mathrm{CI}=1.92-9.86)$, and complication rate $(\mathrm{OR}=0.28,95 \% \mathrm{CI}$ $=0.15-0.55)$, but not for RRFF-C $(\mathrm{OR}=0.79,95 \% \mathrm{CI}=0.52-1.20)$. Between 1998-2004, RRFF-C was correlated with tumor number, pathological stage, DM presence, presence of complication, CIS detection and surgeon experience. After the introduction of the teaching program, only tumor number, DM presence and surgeon experience influenced the RRFF-C.

Conclusion: Our findings suggest the hypothesis that the teaching program might have an impact of quality of TURBT, but it failed to improve the RRFF-C.
\end{abstract}

Key Words: Urinary bladder neoplasms, Cystoscopic surgical procedure, Quality control, Neoplasm local recurrence, Learning curve

\section{INTRODUCTION}

transurethral resection for bladder tumor (TURBT) is considered the gold standard for the treatment of primary and recurrent non-muscle-invasive bladder cancer (NMIBC). ${ }^{[1]}$ The main goals of TURBT are diagnosis (the adequate endoscopic assessment of number, size, aspect, and location of

\footnotetext{
*Correspondence: Massimo Lazzeri; Email: massimo.lazzeri@humanitas.it; Address: Department of Urology, Istituto clinico Humanitas IRCCS, Humanitas Clinical and Research Center, via Manzoni 56, 20089, Rozzano (Milan), Italy.
} 
the bladder tumors) and the therapeutic removal of the whole lesion with the detection of carcinoma in situ (CIS). ${ }^{[1-4]}$

Attempts have been made to measure outcomes and to check the quality of several surgical and urological procedures. ${ }^{[2,5]}$ It is important that TURBT not be excluded from these quality assessments since complications, early recurrence and inadequate pathological staging may result from a badly performed procedure. A review of seven randomized controlled studies, performed by the European organization for research and treatment of cancer (EORTC) in 2012, showed significant inter-institutional variability in the RRFF-C which was attributed to variability in the quality of the resections. ${ }^{[6]}$ Importantly, several quality outcome measures are considered for TURBT, including the presence/absence of detrusor muscle (DM) in the first TURBT, the detection of CIS and the intraoperative or post-operative complication rate. In a preliminary study with a relatively small sample a supervised TURBT teaching program was shown to have an impact on the quality of TURBT. ${ }^{[7]}$ The 3-month recurrence rate decreased from $28 \%$ to $16 \%$ and the presence of DM increased from $50 \%$ to $84 \%$ regardless of surgeon experience. In order to assess the clinical impact of a teaching program aimed at improving the outcome of TURBT, we tested the hypothesis that the introduction of such a program could improve the presence of DM and the detection of CIS as well as result in a reduction of complications and recurrences.

\section{MATERIAL AND METHODS}

This is an observational retrospective cohort study of prospectively recorded data from our Institutional database that was authorized by our Institutional Ethical Review Board. Patients with a first clinical diagnosis of NMIBC, scheduled for TURBT at our tertiary high-volume hospital were considered eligible for the study. The Strengthening the Reporting of Observational Studies in Epidemiology (STROBE) recommendations were followed in drafting and reporting data. ${ }^{[8]}$

Male and female patients with a first diagnosis of NMIBC, aged $>18$ years, who underwent TURBT with radical intent, and who consented to the collection and use of their data for clinical research purposes were considered eligible for this cohort analysis. The following exclusion criteria were applied: un-complete chart data, patients with tumors that were clinically considered to be muscle invasive, patients with radiographic images suggestive of muscle-invasive disease, patients who did not attend the follow-up cystoscopy after 3 months and patients with non-transitional cell carcinoma.

TURBT was performed according to the following standard steps: resection of the exophytic part of the tumor (first specimen), deep resection of the detrusor muscle (second

Published by Sciedu Press specimen) and any possible cold cup biopsy of the tumor base, including the muscle (third specimen). All patients without perforation or significant bleeding were scheduled to receive an early single instillation of chemotherapeutic agents (Mitomycin C).

In 2005 we introduced a systematic TURBT teaching program for assisting surgeons in improving their performance. It included several novelties, which had not been previously standardized. All patients underwent a flexible cystoscopy and a bladder diagram preoperatively in order to identify and localize tumors in the bladder. A video-TURBT archive was established and the cases were reviewed. Surgeons were classified as "consultants" (seniors with $>5$ years of training and more than 100 previously performed TURBT) vs. "residents" (trainees with $<5$ years of training and less than 100 previously performed TURBT). ${ }^{[9]}$ For resident surgeons, the regular presence of a consultant urologist in the operating room to act as a tutor was introduced. The tutor supervised the TURBT performance and ensured a correct adherence to the guidelines. Finally, routine weekly multidisciplinary discussion meetings between urologists and genito-urinary pathologists were held. Every case was discussed in the presence of surgeons who were in the operating room. The discussion addressed the following points: the pathological report, the procedure performed, adherence to the guidelines, complications and the need for adjuvant intravesical treatments. For the purpose of our analysis patients with complete chart data were split into two groups: those patients who underwent TURBT in the years 1998-2004, when no tutoring or systematic teaching approach was available in our department, and those who underwent TURBT in the years 2005-2010 after the introduction of the teaching program.

The outcomes of interest were the presence/absence of DM in the specimen, the CIS detection rate, the complication rate (bladder perforation or peri-postoperative bleeding defined intraoperatively, but not post-operative complications as defined by Clavien-Dindo classification scale) and recurrence rate at the first follow-up cystoscopy (RRFF-C) (also including disease persistence 3 months post-operatively). ${ }^{[4]}$ Histologic analysis of all tumor samples was based on the 1973 World Health Organization (WHO) classification and on the $2004 \mathrm{WHO} / \mathrm{ISUP}$ classification and was performed by experienced uropathologists. ${ }^{[10]}$

\section{Statistical analysis}

Data are described as numbers and percentages, or means and standard deviations, as appropriate. Pre and post-program data, as well as surgeon data, were compared using the chi square test with Fisher correction when necessary. We performed a univariable logistic regression to assess the impact 
of potential predictor variables on the outcomes of interest for each period (1998-2004 vs. 2005-2010). Multivariable logistic regression of the whole series was performed for each quality outcome in order to assess the predictive power of the teaching program on TURBT outcomes. The analysis was repeated for the RRFF-C outcome. A $P<.05$ was considered significant. All analyses were done with Stata13.

\section{RESUlts}

The final cohort consisted of 427 patients (complete data on charts): 199 treated during the period 1998-2004, and 228 during the period 2005-2010. Demographic and clinical characteristics of patients are described in Table 1. Fifty-two patients with perforation or bleeding did not receive early intravesical treatment. Overall, 78 patients were classified as low-risk and did not receive any adjuvant treatment, $225 \mathrm{had}$ an intermediate-risk and underwent Mitomycin C (MMC) while 124 with high risk received BCG. Considering both time periods, consultant surgeons performed $60.2 \%$ of all TURBT procedures. Comparing the data from 1998-2004 to that from 2005-2010, the incidence of DM in the specimen increased from $59.3 \%$ to about $82 \%(P<.001)$, CIS detection increased from $6 \%$ to $15.4 \%(P=.003)$, and the complication rate (clinically relevant perforation and/or bleeding) decreased from $18.6 \%$ to $6.6 \%(P<.001)$. The RRFF-C decreased by $31.7 \%$ to $26.8 \%$, but this was not statistically significant $(P=.286)($ see Table 1$)$.

Table 1. Patient demographics, tumor characteristics, and surgeon category at the time of first TURBT for NMIBC

\begin{tabular}{|c|c|c|c|c|}
\hline & All & Years (1998-2004) & Years (2005-2010) & $\boldsymbol{P}$ \\
\hline Patients number & 427 & 199 & 228 & \\
\hline Gender (F) & $121(28.3 \%)$ & $57(28.6 \%)$ & $64(28.1 \%)$ & .915 \\
\hline Tumor size $(>3 \mathrm{~cm})$ & $93(21.8 \%)$ & $45(22.6 \%)$ & $48(21.1 \%)$ & .725 \\
\hline Tumor multiplicity (multiple) & $114(26.7 \%)$ & $46(23.1 \%)$ & $68(29.8 \%)$ & .126 \\
\hline Primary Grade* (G3) & $117(27.4 \%)$ & $51(25.6 \%)$ & $66(29.0 \%)$ & .449 \\
\hline Primary Stage (T1) & $107(25.1 \%)$ & $49(24.6 \%)$ & $58(25.4 \%)$ & .911 \\
\hline CIS detection & $47(11.0 \%)$ & $12(6.0 \%)$ & $35(15.4 \%)$ & .003 \\
\hline Detrusor muscle & 305 (71.4\%) & $118(59.3 \%)$ & $187(82.0 \%)$ & $<.001$ \\
\hline $\begin{array}{l}\text { Clinically relevant complication (bleeding } \\
\text { and/or perfusion) }\end{array}$ & $52(12.2 \%)$ & $37(18.6 \%)$ & $15(6.6 \%)$ & $<.001$ \\
\hline Surgeon category (Senior) & $257(60.2 \%)$ & $124(62.3 \%)$ & $133(58.3 \%)$ & .429 \\
\hline RRFF-C & $124(29.0 \%)$ & $63(31.7 \%)$ & $61(26.8 \%)$ & .286 \\
\hline
\end{tabular}

Note. "WHO 1973.

\subsection{Impact of the teaching program}

Univariable analysis showed that the teaching program was associated with $\mathrm{DM}$ presence $(\mathrm{OR}=3.13,95 \% \mathrm{CI}=2.01$ 4.86), CIS detection $(\mathrm{OR}=2.83,95 \% \mathrm{CI}=1.42-5.61)$ and complication rate $(\mathrm{OR}=0.31,95 \% \mathrm{CI}=0.16-0.58)$; no association was found for RRFF-C $(\mathrm{OR}=0.79,95 \% \mathrm{CI}=$ $0.52-1.20)$. The statistical analysis was complemented with multivariable analysis including the following variables: resident/senior, tumor size and number, pathological grade and stage and teaching. Multivariate logistic regression analysis on the whole group of patients with the teaching program as an independent prognostic factor revealed that it had a favorable impact on $\mathrm{DM}$ presence rate $(\mathrm{OR}=3.92,95 \% \mathrm{CI}$ $=2.42-6.33)$, CIS detection rate $(\mathrm{OR}=4.36,95 \% \mathrm{CI}=1.92$ $9.86)$ and complication rate $(\mathrm{OR}=0.30,95 \% \mathrm{CI}=0.16-0.56)$.

\subsection{RRFF-C: an analysis stratified by time}

During the period 1998-2004, the univariable analysis showed that RRFFC was associated with tumor number (OR $=3.19,95 \% \mathrm{CI}=1.61-6.31, P=.001)$ stage $(\mathrm{OR}=3.89$, 98\% CI $=1.98-7.64, P<.001), \mathrm{DM}$ presence $(\mathrm{OR}=0.41$,
95\% CI $=0.22-0.75, P=.004)$ and CIS detection $(\mathrm{OR}=2.91$, $95 \% \mathrm{CI}=1.45-5.81, P=.001)$. After the introduction of the teaching program only tumor number $(\mathrm{OR}=4.02,95 \% \mathrm{CI}=$ $2.15-7.49, P=.001)$ and $\mathrm{DM}$ presence $(\mathrm{OR}=0.07,95 \% \mathrm{CI}=$ 0.03-0.16, $P<.001)$ were associated with RRFF-C.

\subsection{Surgeon experience: analysis stratified by time}

Consultant surgeons had better performance rates compared to resident surgeons in both periods but, as can be seen from Table 2, the teaching program had a positive effect on both groups. Both resident and consultant surgeons had fewer procedures without DM following the introduction of the teaching program: 41 (54.7\%) vs. 24 (25.3\%) for resident surgeons $(P<.001)$ and $40(32.3 \%)$ vs. $17(12.8 \%)$ for consultant surgeons $(P<.001)$. Furthermore, in the years with the teaching program both consultant and resident surgeons had fewer RRFF-C, even if not statistically significant: 33 (44.0\%) vs. $37(39.0 \%)$ for resident surgeons $(P=.533)$ and $30(24.2 \%)$ vs. $24(18.1 \%)$ for consultant surgeons $(P=$ $.284)$.

ISSN 1925-4067 E-ISSN 1925-4075 
Table 2. Association of surgeon category and tumor grade, stage, and DM in NMIBC in the two period: 1998-2004 and 2005-2010

\begin{tabular}{|c|c|c|c|c|c|c|}
\hline & \multicolumn{3}{|l|}{ 1998-2004 } & \multicolumn{3}{|l|}{ 2005-2010 } \\
\hline & Resident & Senior & $P$ & Resident & Senior & $\boldsymbol{P}$ \\
\hline Patients number & 75 & 124 & & 95 & 133 & \\
\hline Gender (F) & $26(34.7 \%)$ & 31 (25.0\%) & .150 & 27 (28.4\%) & 37 (27.8\%) & 1.000 \\
\hline Size $(>3 \mathrm{~cm})$ & $25(33.3 \%)$ & $20(16.1 \%)$ & .008 & $26(27.4 \%)$ & $22(16.5 \%)$ & .069 \\
\hline Tumor number (Multiple) & $20(26.7 \%)$ & $26(21.0 \%)$ & .388 & $25(26.3 \%)$ & 43 (32.3\%) & .379 \\
\hline Tumor grade (3) & $22(29.3 \%)$ & $29(23.4 \%)$ & .403 & $20(21.1 \%)$ & 46 (34.6\%) & .027 \\
\hline Tumor stage (T1) & $19(25.3 \%)$ & 30 (24.2\%) & .867 & $14(14.7 \%)$ & $44(33.1 \%)$ & .002 \\
\hline DM (present) & $34(45.3 \%)$ & $84(67.7 \%)$ & .003 & $71(74.7 \%)$ & $116(87.2 \%)$ & .022 \\
\hline RRFF-C (present) & $33(44.0 \%)$ & $30(24.2 \%)$ & .005 & 37 (39.0\%) & 24 (18.1\%) & .001 \\
\hline
\end{tabular}

\section{Discussion}

Our findings demonstrate that the introduction of a teaching program is an independent outcome predictor factor and may significantly improve the quality of TURBT. It has a positive effect both on resident and consultant staff surgeons. On the contrary, no significant improvement of RRFF-C was found at cystoscopy 3 months post-TURBT.

The TURBT still represents the most frequently performed surgical approach in every urologic unit and provides clinical outcomes of paramount importance from both an oncologic and economic viewpoint. ${ }^{[11,12]}$ The expected results of TURBT include the complete eradication and an adequate pathological staging and grading of NMIBC. The procedure is generally assumed to be technically easy to complete after a short learning curve and is frequently proposed to residents as the first surgical approach during their urologic training. Unfortunately, the literature shows that the quality of TURBT may be low. ${ }^{[9]}$ Such data are registered in peripheral as well as reference and academic centers. ${ }^{[13]}$ The reasons for these impressively low-quality TURB outcomes are certainly numerous and probably have different impacts in different clinical contexts. Adiyat et al. emphasized the role of an accurate pre-operative examination of the prostatic urethra and bladder (by using 12-degree and 30 to 70-degree optical lenses, respectively). ${ }^{[14]}$ Additionally, the use of a video camera to provide adequate optical magnification and the separate collection of specimens taken from the exophytic part of the tumor and it base, including the detrusor muscle, have been strongly suggested. Pan and Soloway supported the use of a transurethral-resection-dedicated operative checklist in order to improve the definition of number, size, location and staging of the tumors. ${ }^{[1]]}$ Finally, the adoption of teaching programs may significantly improve outcomes. ${ }^{[7]}$

Brausi et al. showed that after the introduction of teaching programs, a $28 \%$ to $16 \%$ decrease of the 3 -month recurrence rate was observed, together with an increase from $50 \%$ to
$84 \%$ for DM detection, regardless of whether the operating surgeon was a consultant or junior. ${ }^{[7]}$ This study, however, which was not published as a full length paper but rather as an abstract, included small sample and showed weaknesses in its statistical analysis as well as. Unfortunately the time dedicated by experienced urologists to train residents to correctly complete a TURB is quite negligible in many urologic units, including academic centers. ${ }^{[15]}$

To date, only a few studies have been designed to assess the presence of DM in TURBT specimens and the rate of RRFF-C after TURBT according to surgeon experience. ${ }^{[4]}$ However there is no shared definition of the surgeon categories (senior, consultant, specialist versus junior, specialist registrar, resident) and/or how to measure experience. Regarding the rate of DM in TURBT specimens, Jesurai et al. reported $45.8 \%$ and $67.3 \%$, and Anderson et al. $62 \%$ and $70 \%$ when procedure were stratified for junior and senior surgeons, respectively. ${ }^{[16,17]}$ The rate of DM presence in our study population was significantly higher after the adoption of the teaching program, going from $45.3 \%$ to $67.7 \%$ in resident surgeons and from $74.7 \%$ to $87.2 \%$ in seniors. This improvement is particularly striking for the resident surgeons, as they reached a quality performance similar to or even better than that reported in the literature. According to Yeo et al. the introduction of a dedicated TURBT checklist and a teaching program involving consultant urologists led to an increase in the presence of DM in the specimens obtained by both the consultant and specialist registrars, ranging from $71 \%$ to $95 \%$ and from $68 \%$ to $89 \%$, respectively. ${ }^{[18]}$ The increase in the rate of DM in TURBT specimens registered in our study for staff member surgeons and residents (59\% and $82 \%$, before and after the teaching program, respectively) is in line with that described by Brausi. ${ }^{[17]}$ On the other hand, the decrease in RRFF-C reported for staff member surgeons was only from $8 \%$ to $3 \%$ following the teaching program introduction. For residents, Brausi reported 28\% RRFF-C before and $16 \%$ after the introduction of the teaching program, 
while in our study the difference was more evident $(46.1 \%$ to $33.4 \%$ ). Likewise, Mariappan et al. described a decrease of RRFFC in $39 \%$ of junior and $25 \%$ of senior surgeons, respectively. ${ }^{[9]}$ Unfortunately, the multivariable analysis failed to show the teaching program as an independent predictor of RR-FFC.

Although our study has several strengths (a large sample size, the multivariable analysis and the setting), it is not without limitations. A primary limitation of the study is that it attributes changes entirely to the "teaching program" and improvements in the surgeon learning curve. As a component of the program included the review of every case with the surgeon and the pathologist, we must consider that this strategy might actually motivate the pathologist to look more carefully for the presence of muscle in the specimen and CIS. The cases spanned a long time period during which both the instrumentation and recommendations for adjuvant treatment changed. We did not consider variables such as patient comorbidity or other confounding factors (smoking-risk factors) in our analysis. The Clavien-Dindo classification was not used for the calculation of complication rate as it was not applied in our Institution throughout the overall period. Although the incidence of postoperative complications is still the most frequently used surrogate marker of quality in surgery, no standard guidelines or criteria exist for reporting surgical complications in the area of urology. In order to close this gap, only in 2012 did an EAU panel attempt to provide recommendations. ${ }^{[20]}$ While the impact of tutors on resident surgeons has been shown, ${ }^{[19]}$ the "reflective techniques", i.e. metacognitive processes that create a greater understanding of the self in performing a procedure, was not used for seniors. ${ }^{[21]}$ Finally we did not report the mid- and long-term oncologic outcomes.

\section{Conclusion}

Our findings confirm and extend previous data on the role of a teaching program in improving the surgical outcome of TURBT. A positive impact of the teaching program on TURBT outcome was observed for DM sampling, CIS detection and complications rate, but not for RRFF-C improvement.

\section{ACKNOWLEDGEMENTS}

We would like to thank Prof. Giovanna Farinelli from Department of Philosophy, Social and Human Sciences and Education, University of Perugia (Italy), who developed and supervised the rationale of our teaching program.

\section{CONFlicts OF INTEREST Disclosure}

The authors declare that there is no conflict of interest statement.

\section{REFERENCES}

[1] Babjuk M, Burger M, Zigeuner R, et al. EAU guidelines on Non-Muscle-invasive urothelial carcinoma of the bladder: Update. Eur Urol. 2013; 64(4): 639-53. PMid:23827737. http://dx.doi .org/10.1016/j. eururo.2013.06.003

[2] Mostafid H, Brausi M. Measuring and improving the quality of transurethral resection for bladder tumour (TURBT). BJU Int. 2012; 109(11): 1579-82. PMid:21992712. http://dx.doi.org/10.11 11/j.1464-410X.2011.10638.x

[3] Mariappan P, Zachou A, Grigor KM, et al. Detrusor muscle in the first, apparently complete transurethral resection of bladder tumour specimen is a surrogate marker of resection quality, predicts risk of early recurrence, and is dependent on operator experience. Eur Urol. 2010; 57(5): 843-9. PMid:19524354. http: $/ / \mathrm{dx}$.doi.org/10.1016/j. eururo.2009.05.047

[4] Mariappan P, Finney SM, Head E, et al. Good quality white-light transurethral resection of bladder tumours (GQ-WLTURBT) with experienced surgeons performing complete resections and obtaining detrusor muscle reduces early recurrence in new non-muscleinvasive bladder cancer: Validation across time and place and recommendation for benchmarking. BJU Int. 2012; 109(11): 1666-73. PMid:22044434. http://dx.doi.org/10.1111/j.1464-410X. 2011.10571.x

[5] Haynes AB, Weiser TG, Berry WR, et al. A surgical safety checklist to reduce morbidity and mortality in a global population. $\mathrm{N} \mathrm{Engl} \mathrm{J}$
Med. 2009; 360(5): 491-9. PMid:19144931. http://dx.doi.org $/ 10.1056 /$ NEJMsa0810119

[6] Brausi M, Collette L, Kurth K, et al. Variability in the recurrence rate at first follow-up cystoscopy after TUR in stage ta T1 transitional cell carcinoma of the bladder: A combined analysis of seven EORTC studies. Eur Urol. 2012; 41(5): 523-31. http: //dx.doi.org/10.1016/S0302-2838(02) 00068-4

[7] Brausi MA, Gavioli M, Peracchia G, et al. Dedicated teaching programs can improve the quality of TUR of non-muscle-invasive bladder tumours (NMIBT): experience of a single institution. Eur Urol. 2008 Suppl; 7(3): 180 (abstract). http://dx.doi.org/10.1016 /s1569-9056(08)60433-9

[8] von Elm E, Altman DG, Egger M, et al. STROBE Initiative. The Strengthening the Reporting of Observational Studies in Epidemiology (STROBE) statement: guidelines for reporting observational studies. Lancet. 2007; 370(9596): 1453-7. http://dx.doi .org/1 $0.1016 / \mathrm{S} 0140-6736(07) 61602-\mathrm{X}$

[9] Mariappan P, Zachou A, Grigor KM. Detrusor muscle in the first transurethral resection of bladder tumour (TURBT) specimen is a surrogate marker of resection quality and is dependant on operator experience. Eur Urol. 2008 Suppl; 7(3): 299 (abstract).

[10] MacLennan GT, Kirkali Z, Cheng L. Histologic Grading of Noninvasive Papillary Urothelial Neoplasms. Eur Urol. 2007; 51(4): 889-98. PMid: 17095142.

[11] Pan D, Soloway MS. The importance of transurethral resection in managing patients with urothelial cancer in the bladder: Proposal for 
a transurethral resection of bladder tumor checklist. Eur Urol. 2012; 61(6): 1199-203. PMid:22464897. http://dx.doi.org/10.1016 /j.eururo.2012.03.018

[12] Richards KA, Smith ND, Steinberg GD. The importance of transurethral resection of bladder tumor in the management of non muscle invasive bladder cancer: A systematic review of novel technologies. J Urol. 2014; 191(6): 1655-64. PMid:24518761. http: //dx.doi.org/10.1016/j.juro.2014.01.087

[13] Gan C, Patel A, Fowler S, et al. Snapshot of transurethral resection of bladder tumours in the United Kingdom Audit (STUKA). BJU Int. 2013; 112(7): 930-5. http://dx.doi.org/10.1111/bju.12235

[14] Adiyat KT, Katkoori D, Soloway CT, et al. Complete transurethral resection of bladder tumor: Are the guidelines being followed? Urology. 2010; 75(2): 365-7. PMid:19963238. http://dx.doi.org/1 $0.1016 /$ j.urology. 2009.08.082

[15] Brausi MA. Challenging the EAU guidelines regarding early repeat transurethral resection. European Urology. 2011 Suppl; 10(3): e5-e7.

[16] Jesurai MG, Harris M, Rogers A, et al. Completeness of first resection of bladder tumor depending on seniority of the surgeon. Eur Urol. 2008 Suppl; 7(3): 138 (abstract).
[17] Anderson CH, Lee H, Stewart S, et al. Early recurrence following photo-dynamic diagnosis (PDD) assisted TURBT is significantly lower than with good quality white light TURBT (GQWL-TURBT) -a prospective controlled study. BJUI. 2010 Suppl; 106(1): 6.

[18] Yeo L, Jain S. Good quality white-light transurethral resection of bladder tumours (GQ-WLTURBT) with experienced surgeons performing complete resections and obtaining detrusor muscle reduces early recurrence in new non-muscle-invasive bladder cancer: validation across time and place and recommendation for benchmarking. BJU Int. 2012; 109(8): E27. PMid:22455408. http: //dx.doi.org/10.1111/j.1464-410X.2012.11011_3.x

[19] Nieder AM, Meinbach DS, Kim SS, et al. Transurethral bladder tumor resection: intraoperative and postoperative complications in a residency setting. J Urol. 2005; 174(6): 2307-9. PMid:16280830. http: //dx.doi.org/10.1097/01.ju.0000181797.19395.03

[20] Mitropoulos D, Artibani W, Graefen M, et al. Reporting and Grading of Complications After Urologic Surgical Procedures: An ad hoc EAU Guidelines Panel Assessment and Recommendations Eur Urol. 2012; 61(2): 341-9.

[21] Aronson L. Twelve tips for teaching reflection at all levels of medical education. Med Teach. 2011; 33(3): 200-5. PMid:20874014. http://dx.doi.org/10.3109/0142159X.2010.507714 